\title{
Singular limits for 2-dimensional elliptic problem involving exponential nonlinearity with nonlinear gradient term
}

\author{
Sami Baraket, Ines Ben Omrane and Taieb Ouni
}

\begin{abstract}
Given a bounded open regular set $\Omega \subset \mathbb{R}^{2}$ and $x_{1}, x_{2}, \ldots, x_{m} \in$ $\Omega$, we give a sufficient condition for the problem

$$
-\operatorname{div}(a(u) \nabla u)=\rho^{2} f(u)
$$

to have a positive weak solution $u$ in $\Omega$ with $u=0$ on $\partial \Omega$, which is singular at each $x_{i}$ as the parameter $\rho$ tends to 0 and under suitable assumptions on exponential functions $a(u)$ and $f(u)$.

Mathematics Subject Classification (2000). 35J60, 53C21, 58J05.

Keywords. Singular limits, Green's function, Nonlinear domain decomposition method.
\end{abstract}

\section{Introduction and statement of the results}

We consider the following problem

$$
\begin{cases}-\operatorname{div}(a(u) \nabla u)=\rho^{2} f(u) & \text { in } \Omega \\ u=0 & \text { on } \partial \Omega,\end{cases}
$$

where $\nabla$ is the gradient symbol and $\Omega$ is an open smooth bounded subset of $\mathbb{R}^{2}$. The function $a(u)$ is assumed to be positive and smooth. The function $f(u)$ is increasing, positive and smooth. The parameter $\rho$ is small. In the following, we take $a(u)=e^{\lambda u}$ and $f(u)=e^{(\lambda+1) u}$ for a small parameter $\lambda>0$ satisfying suitable assumption which will be fixed later on. Then problem (1) takes the form

$$
\begin{cases}-\Delta u-\lambda|\nabla u|^{2}=\rho^{2} e^{u} & \text { in } \Omega \\ u=0 & \text { on } \partial \Omega .\end{cases}
$$

Using the transformation

$$
w=\left(\lambda \rho^{2} e^{u}\right)^{\lambda}
$$


then the function $w$ satisfies the problem

$$
\begin{cases}-\Delta w=w^{\frac{\lambda+1}{\lambda}} & \text { in } \Omega \\ w=\left(\lambda \rho^{2}\right)^{\lambda} & \text { on } \partial \Omega .\end{cases}
$$

Problem (3) has been studied by Ren and Wei in [13], since the exponent $q=\frac{\lambda+1}{\lambda}$ tends to infinity as $\lambda$ tends to 0 .

We denote by $\varepsilon$ the smallest positive parameter satisfying

$$
\rho^{2}=\frac{8 \varepsilon^{2}}{\left(1+\varepsilon^{2}\right)^{2}} .
$$

Remark that $\rho \sim \varepsilon$ as $\varepsilon \longrightarrow 0$.

We will suppose in the following

$(A): \quad$ If $0<\varepsilon<\lambda$, then $\lambda^{1+\delta / 2} \varepsilon^{-\delta} \longrightarrow 0$ as $\lambda \longrightarrow 0$, for any $\delta \in(0,1)$.

In particular, if we take $\lambda=\mathcal{O}\left(\varepsilon^{2 / 3}\right)$, then condition $(A)$ is satisfied. With assumption $(A)$, we can treat Eq. (2) as a perturbation of the Liouville equation

$$
-\Delta u=\rho^{2} e^{u} \quad \text { in } \quad \Omega \subset \mathbb{R}^{2} .
$$

We look for a sequence of solutions $u_{\rho, \lambda}$ of (2) which converges to some non trivial singular function on some set as the parameters $\rho$ and $\lambda$ tend to 0 . The presence of the gradient term can have significant influence on the existence of a solution, as well as on its asymptotic behavior. Problem (2) with the condition $u_{\mid \partial \Omega}=0$ replaced by $u_{\mid \partial \Omega}=+\infty$ arises from many branches of mathematics and applied mathematics, and has been discussed by many authors and in many contexts, see, e.g. [1,6-9,11,12,15,21].

Given $\lambda=0$, then solutions of problem (2) solve

$$
\begin{cases}-\Delta u=\rho^{2} e^{u} & \text { in } \Omega \\ u=0 & \text { on } \partial \Omega,\end{cases}
$$

where $\Omega$ is a domain of $\mathbb{R}^{2}$. The study of this equation goes back to 1853 when Liouville derived a representation formula for all solutions of (5) which are defined in $\mathbb{R}^{2}$, see [10]. It turns out that, beside the applications in geometry, elliptic equations with exponential nonlinearity also arise in modeling many physical phenomena such as thermionic emission, isothermal gas sphere, gas combustion, gauge theory $[16], \ldots$

When $\rho$ tends to 0 , the asymptotic behavior of nontrivial branches of solutions of (5) is well understood thanks to the pioneer work of Suzuki [14] which characterizes the possible limit of nontrivial branches of solutions of (5). The existence of nontrivial branches of solutions was first proved by Weston [19] and then a general positive result for problem (5) has been obtained by Baraket and Pacard [4]. These results were extended, applying to the ChernSimons vortex theory in mind, by Esposito et al. [6] and Del Pino et al. [5] to handle equations of the form

$$
-\Delta u=\rho^{2} V e^{u},
$$

where $V$ is a non constant (positive) function. Let us also mention that the construction of nontrivial branches of solutions of semilinear equations with 
exponential nonlinearities allowed Wente [17] to provide a counter example to a conjecture of Hopf concerning the existence of compact (immersed) constant mean curvature surfaces in Euclidean space, see also $[18,20]$.

We introduce the Green's function $G\left(x, x^{\prime}\right)$ defined on $\Omega \times \Omega$, to be the solution of

$$
\begin{cases}-\Delta G\left(x, x^{\prime}\right)=8 \pi \delta_{x=x^{\prime}} & \text { in } \Omega \\ G\left(x, x^{\prime}\right)=0 & \text { on } \partial \Omega\end{cases}
$$

and let its regular part

$$
H\left(x, x^{\prime}\right)=G\left(x, x^{\prime}\right)+4 \log \left|x-x^{\prime}\right| .
$$

Let $m \in \mathbb{N}$, we set

$$
\mathcal{F}\left(x_{1}, \ldots, x_{m}\right)=\sum_{j=1}^{m} H\left(x_{j}, x_{j}\right)+\sum_{i \neq j} G\left(x_{i}, x_{j}\right)
$$

which is well defined in $\Omega^{m}$ for $x_{i} \neq x_{j}$ if $i \neq j$.

Our main result is the following:

Theorem 1. Given $\beta \in(0,1)$. Let $\Omega$ be an open smooth bounded set of $\mathbb{R}^{2}$, $\lambda>0$ satisfying condition $(A)$ and $S=\left\{x_{1}, \ldots, x_{m}\right\} \subset \Omega$ be a non empty set. Assume that $\left(x_{1}, \ldots, x_{m}\right)$ is a nondegenerate critical point of the function

$$
\mathcal{F}\left(x_{1}, \ldots, x_{m}\right)=\sum_{j=1}^{m} H\left(x_{j}, x_{j}\right)+\sum_{i \neq j} G\left(x_{i}, x_{j}\right) \quad \text { in }(\Omega)^{m},
$$

then there exist $\rho_{0}>0, \lambda_{0}>0$ and a family $\left\{u_{\rho, \lambda}\right\}_{\substack{0<\rho<\rho_{0} \\ 0<\lambda<\lambda_{0}}}$ of solutions of (2), such that

$$
\lim _{\substack{\rho \rightarrow 0 \\ \lambda \rightarrow 0}} u_{\rho, \lambda}=\sum_{j=1}^{m} G\left(x_{j}, \cdot\right)
$$

in $\mathcal{C}_{\text {loc }}^{2, \beta}\left(\Omega-\left\{x_{1}, \ldots, x_{m}\right\}\right)$.

Our result reduces the study of nontrivial branches of solutions of (2) to the search for critical points of the function $\mathcal{F}$ defined in $(6)$. Observe that the assumption on the nondegeneracy of the critical point is a rather mild assumption since it is certainly fulfilled for generic choice of the open domain $\Omega$.

One of the purposes of the present paper is to present a rather efficient method to solve such singularly perturbed problems. This method has already been used successfully in geometric context (constant mean curvature surfaces, constant scalar curvature metrics, extremal Kähler metrics, manifolds with special holonomy, ... ). It also appeared for the first time in the work [3] in the context of partial differential equations.

\section{Construction of the approximate solution}

We first describe the rotationally symmetric approximate solutions of

$$
-\Delta u-\lambda|\nabla u|^{2}=\rho^{2} e^{u}
$$


in $\mathbb{R}^{2}$ which will play a central role in our analysis. Given $\varepsilon>0$, we define

$$
u_{\varepsilon}(x):=2 \log \left(1+\varepsilon^{2}\right)-2 \log \left(\varepsilon^{2}+|x|^{2}\right)
$$

which is clearly a solution of

$$
-\Delta u=\rho^{2} e^{u}
$$

in $\mathbb{R}^{2}$.

Let us notice that Eqs. (7) and (9) are invariant under dilation in the following sense: If $v$ is a solution of (7) or (9) and if $\tau>0$, then $v(\tau \cdot)+2 \log \tau$ is also a solution of (7) or (9). With this observation in mind, we define for all $\tau>0$

$$
u_{\varepsilon, \tau}(x):=2 \log \left(1+\varepsilon^{2}\right)+2 \log \tau-2 \log \left(\varepsilon^{2}+|\tau x|^{2}\right) .
$$

\subsection{A linearized operator on $\mathbb{R}^{2}$}

For all $\varepsilon, \tau, \lambda>0$, we define

$$
R_{\varepsilon, \lambda}:=\tau r_{\varepsilon, \lambda} / \varepsilon
$$

where

$$
r_{\varepsilon, \lambda}:=\max (\sqrt{\varepsilon}, \sqrt{\lambda})
$$

We define the linear second order elliptic operator

$$
\mathbb{L}:=-\Delta-\frac{8}{\left(1+|x|^{2}\right)^{2}}
$$

which corresponds to the linearization of (9) about the solution $u_{1}\left(=u_{\varepsilon=\tau=1}\right)$ which has been defined in the previous section.

We are interested in the classification of bounded solutions of $\mathbb{L} w=0$ in $\mathbb{R}^{2}$. Some solutions are easy to find. For example, we can define

$$
\phi_{0}(x):=\frac{r}{2} \partial_{r} u_{1}(x)+1=2 \frac{1-r^{2}}{1+r^{2}},
$$

where $r=|x|$. Clearly $\mathbb{L} \phi_{0}=0$ and this reflects the fact that (9) is invariant under the group of dilations $\tau \longrightarrow u(\tau \cdot)+2 \log \tau$. We also define, for $i=1,2$

$$
\phi_{i}(x):=-\partial_{x_{i}} u_{1}(x)=\frac{2 x_{i}}{1+|x|^{2}},
$$

which are also solutions of $\mathbb{L} \phi_{i}=0$ since these solutions correspond to the invariance of the equation under the group of translations $a \longrightarrow u(\cdot+a)$.

We recall the following result which classifies all bounded solutions of $\mathbb{L} w=0$ which are defined in $\mathbb{R}^{2}$.

Lemma 1. [4] Any bounded solution of $\mathbb{L} w=0$ defined in $\mathbb{R}^{2}$ is a linear combination of $\phi_{i}$ for $i=0,1,2$.

Let $B_{r}$ denotes the ball of radius $r$ centered at the origin in $\mathbb{R}^{2}$. 
Definition 1. Given $k \in \mathbb{N}, \beta \in(0,1)$ and $\mu \in \mathbb{R}$, we introduce the Hölder weighted space $\mathcal{C}_{\mu}^{k, \beta}\left(\mathbb{R}^{2}\right)$ as the space of functions $w \in \mathcal{C}_{l o c}^{k, \beta}\left(\mathbb{R}^{2}\right)$ for which the following norm

$$
\|w\|_{\mathcal{C}_{\mu}^{k, \beta}\left(\mathbb{R}^{2}\right)}:=\|w\|_{\mathcal{C}^{k, \beta}\left(\bar{B}_{1}\right)}+\sup _{r \geq 1}\left(\left(1+r^{2}\right)^{-\mu / 2}\|w(r \cdot)\|_{\mathcal{C}^{k, \beta}\left(\bar{B}_{1}-B_{1 / 2}\right)}\right)
$$

is finite.

We also define

$$
\mathcal{C}_{\text {rad, }, \mu}^{k, \beta}\left(\mathbb{R}^{2}\right)=\left\{f \in \mathcal{C}_{\mu}^{k, \beta}\left(\mathbb{R}^{2}\right) ; f(x)=f(|x|), \forall x \in \mathbb{R}^{2}\right\} .
$$

As a consequence of the result of Lemma 1, we recall the surjectivity result of $\mathbb{L}$ given in [4].

Proposition 1. [4]

(i) Assume that $\mu>1$ and $\mu \notin \mathbb{N}$, then

$$
\begin{aligned}
L_{\mu}: \mathcal{C}_{\mu}^{2, \beta}\left(\mathbb{R}^{2}\right) & \longrightarrow \mathcal{C}_{\mu-2}^{0, \beta}\left(\mathbb{R}^{2}\right) \\
w & \longmapsto \mathbb{L} w
\end{aligned}
$$

is surjective.

(ii) Assume that $\delta>0$ and $\delta \notin \mathbb{N}$ then

$$
\begin{aligned}
L_{\delta}: \mathcal{C}_{\text {rad }, \delta}^{2, \beta}\left(\mathbb{R}^{2}\right) & \longrightarrow \mathcal{C}_{\text {rad, }, \delta-2}^{0, \beta}\left(\mathbb{R}^{2}\right) \\
w & \longmapsto \mathbb{L} w
\end{aligned}
$$

is surjective.

We set $\bar{B}_{1}^{*}=\bar{B}_{1}-\{0\}$.

Definition 2. Given $k \in \mathbb{N}, \beta \in(0,1)$ and $\mu \in \mathbb{R}$, we introduce the Hölder weighted space $\mathcal{C}_{\mu}^{k, \beta}\left(\bar{B}_{1}^{*}\right)$ as the space of functions in $\mathcal{C}_{\text {loc }}^{k, \beta}\left(\bar{B}_{1}^{*}\right)$ for which the following norm

$$
\|u\|_{\mathcal{C}_{\mu}^{k, \beta}\left(\bar{B}_{1}^{*}\right)}=\sup _{r \leq 1 / 2}\left(r^{-\mu}\|u(r \cdot)\|_{\mathcal{C}^{k, \beta}\left(\bar{B}_{2}-B_{1}\right)}\right)
$$

is finite.

Then we define the subspace of radial functions in $\mathcal{C}_{\text {rad, }, \delta}^{k, \beta}\left(\bar{B}_{1}^{*}\right)$ by

$$
\mathcal{C}_{\text {rad, }, \delta}^{k, \beta}\left(\bar{B}_{1}^{*}\right)=\left\{f \in \mathcal{C}_{\delta}^{k, \beta}\left(\mathbb{R}^{2}\right) ; f(x)=f(|x|), \forall x \in \bar{B}_{1}^{*}\right\} .
$$

We would like to find a solution $u$ of

$$
\Delta u+\lambda|\nabla u|^{2}+\rho^{2} e^{u}=0
$$

in $\bar{B}_{r_{\varepsilon, \lambda}}$. Using the transformation

$$
v(x)=u\left(\frac{\varepsilon}{\tau} x\right)+4 \log \varepsilon-2 \log \left(\tau\left(1+\varepsilon^{2}\right) / 2\right),
$$

then Eq. (13) is equivalent to

$$
\Delta v+\lambda|\nabla v|^{2}+2 e^{v}=0
$$

in $\bar{B}_{R_{\varepsilon, \lambda}}$. Now we look for a solution of (14) of the form

$$
v(x)=u_{1}(x)+h(x),
$$


this amounts to solve

$$
\mathbb{L} h=\frac{8}{\left(1+|x|^{2}\right)^{2}}\left(e^{h}-h-1\right)+\lambda\left|\nabla\left(u_{1}+h\right)\right|^{2}
$$

in $\bar{B}_{R_{\varepsilon, \lambda}}$.

We will need the following definition.

Definition 3. Given $\bar{r} \geq 1, k \in \mathbb{N}, \beta \in(0,1)$ and $\mu \in \mathbb{R}$, the weighted space $\mathcal{C}_{\mu}^{k, \beta}\left(B_{\bar{r}}\right)$ is defined to be the space of functions $w \in \mathcal{C}^{k, \beta}\left(B_{\bar{r}}\right)$ endowed with the norm

$$
\|w\|_{\mathcal{C}_{\mu}^{k, \beta}\left(\bar{B}_{\bar{r})}\right.}:=\|w\|_{\mathcal{C}^{k, \beta}\left(B_{1}\right)}+\sup _{1 \leq r \leq \bar{r}}\left(r^{-\mu}\|w(r \cdot)\|_{\mathcal{C}^{k, \beta}\left(\bar{B}_{1}-B_{1 / 2}\right)}\right) .
$$

For all $\sigma \geq 1$, we denote by

$$
\mathcal{E}_{\sigma}: \mathcal{C}_{\mu}^{0, \beta}\left(\bar{B}_{\sigma}\right) \longrightarrow \mathcal{C}_{\mu}^{0, \beta}\left(\mathbb{R}^{2}\right)
$$

the extension operator defined by

$$
\mathcal{E}_{\sigma}(f)(x)= \begin{cases}f(x) & \text { for }|x| \leq \sigma \\ \chi\left(\frac{|x|}{\sigma}\right) f\left(\sigma \frac{x}{|x|}\right) & \text { for }|x| \geq \sigma,\end{cases}
$$

where $t \longmapsto \chi(t)$ is a smooth nonnegative cutoff function identically equal to 1 for $t \leq 1$ and identically equal to 0 for $t \geq 2$. It is easy to check that there exists a constant $c=c(\mu)>0$, independent of $\sigma \geq 1$, such that

$$
\left\|\mathcal{E}_{\sigma}(w)\right\|_{\mathcal{C}_{\mu}^{0, \beta}\left(\mathbb{R}^{2}\right)} \leq c\|w\|_{\mathcal{C}_{\mu}^{0, \beta}\left(\bar{B}_{\sigma}\right)} .
$$

We fix

$$
\delta \in(0,1)
$$

and denote by $\mathcal{G}_{\delta}$ to be a right inverse of $\mathbb{L}_{\delta}$ provided by Proposition 1 . To find a solution of (15) it is enough to find a fixed point $h$, in a small ball of $\mathcal{C}_{\text {rad, } \delta}^{2, \beta}\left(\mathbb{R}^{2}\right)$, solution of

$$
h=\aleph(h)
$$

where

$$
\aleph(h):=\mathcal{G}_{\delta} \circ \mathcal{E}_{\delta} \circ \mathfrak{R}(h)
$$

with

$$
\mathfrak{R}(h)=\frac{8}{\left(1+|x|^{2}\right)^{2}}\left(e^{h}-h-1\right)+\lambda\left|\nabla\left(u_{1}+h\right)\right|^{2} .
$$

We have

$$
|\Re(0)|=\lambda\left|\nabla u_{1}\right|^{2} .
$$

This implies that given $\kappa>0$, there exists $c_{\kappa}>0$ (which can depend only on $\kappa)$, such that for $\delta \in(0,1)$ and $|x|=r$, we have

$$
\sup _{r \leq R_{\varepsilon, \lambda}} r^{2-\delta}|\Re(0)| \leq \sup _{r \leq R_{\varepsilon, \lambda}} r^{2-\delta} \lambda\left|\nabla u_{1}\right|^{2} \leq c_{\kappa} \lambda .
$$

Then

$$
\|\aleph(0)\|_{C_{r a d, \delta}^{2, \beta}} \leq c_{\kappa} r_{\varepsilon, \lambda}^{2}
$$


Making use of Proposition 1 together with (17), we conclude that

$$
\|h\|_{\mathcal{C}_{\text {rad }, \delta}^{2, \beta}\left(\mathbb{R}^{2}\right)} \leq 2 c_{\kappa} r_{\varepsilon, \lambda}^{2} .
$$

Now, let $h_{1}, h_{2}$ in $B\left(0,2 c_{\kappa} r_{\varepsilon, \lambda}^{2}\right)$ of $\mathcal{C}_{\text {rad, }, \delta}^{2, \beta}\left(\mathbb{R}^{2}\right)$, for $\delta \in(0,1)$, then

$$
\begin{aligned}
\sup _{r \leq R_{\varepsilon, \lambda}} & r^{2-\delta}\left|\mathfrak{R}\left(h_{2}\right)-\mathfrak{R}\left(h_{1}\right)\right| \\
\leq & c_{\kappa} \sup _{r \leq R_{\varepsilon, \lambda}} r^{2-\delta}\left(1+|x|^{2}\right)^{-2}\left|e^{h_{2}}-e^{h_{1}}+h_{1}-h_{2}\right| \\
& +c_{\kappa} \lambda \sup _{r \leq R_{\varepsilon, \lambda}} r^{2-\delta}\left(\left|\nabla\left(u_{1}+h_{2}\right)\right|^{2}-\left|\nabla\left(u_{1}+h_{1}\right)\right|^{2}\right) \\
\leq & c_{\kappa} \sup _{r \leq R_{\varepsilon, \lambda}} r^{-2-\delta}\left|h_{2}-h_{1}\right|\left|h_{2}+h_{1}\right| \\
& +c_{\kappa} \lambda \sup _{r \leq R_{\varepsilon, \lambda}} r^{2-\delta}\left|\nabla\left(h_{2}-h_{1}\right)\right|\left(\left|\nabla\left(h_{2}+h_{1}\right)\right|+2\left|\nabla u_{1}\right|\right) \\
\leq & c_{\kappa} r_{\varepsilon, \lambda}^{2}\left\|h_{2}-h_{1}\right\|_{\mathcal{C}_{r a d, \delta}^{2, \beta}\left(\mathbb{R}^{2}\right)}+c_{\kappa} r_{\varepsilon, \lambda}^{2}\left\|h_{2}-h_{1}\right\|_{\mathcal{C}_{r a d, \delta}^{2, \beta}\left(\mathbb{R}^{2}\right)} .
\end{aligned}
$$

Similarly, making use of Proposition 1 together with (17), we conclude that given $\kappa>0$, there exist $\varepsilon_{\kappa}>0, \lambda_{\kappa}>0$ and $\bar{c}_{\kappa}>0$ (only depend on $\kappa$ ) such that

$$
\left\|\aleph\left(h_{2}\right)-\aleph\left(h_{1}\right)\right\|_{\mathcal{C}_{\text {rad, }, \delta}^{2, \beta}\left(\mathbb{R}^{2}\right)} \leq \bar{c}_{\kappa} r_{\varepsilon, \lambda}^{2}\left\|h_{2}-h_{1}\right\|_{\mathcal{C}_{\text {rad }, \delta}^{2, \beta}\left(\mathbb{R}^{2}\right)} .
$$

Reducing $\varepsilon_{\kappa}>0$ and $\lambda_{\kappa}>0$ if necessary, we can assume that,

$$
\bar{c}_{\kappa} r_{\varepsilon, \lambda}^{2} \leq \frac{1}{2}
$$

for all $\lambda \in\left(0, \lambda_{\kappa}\right)$ and $\varepsilon \in\left(0, \varepsilon_{\kappa}\right)$. Then, (19) and (20) are enough to show that

$$
h \longmapsto \aleph(h)
$$

is a contraction from the ball

$$
\left\{h \in \mathcal{C}_{\text {rad }, \delta}^{2, \beta}\left(\mathbb{R}^{2}\right):\|h\|_{\mathcal{C}_{\text {rad, }}^{2, \beta}\left(\mathbb{R}^{2}\right)} \leq 2 c_{\kappa} r_{\varepsilon, \lambda}^{2}\right\}
$$

into itself and hence has a unique fixed point $h$ in this set. This fixed point is a solution of (18) in $\bar{B}_{R_{\varepsilon, \lambda}}$. We summarize this in the following proposition.

Proposition 2. Given $\kappa>0$. There exist $\varepsilon_{\kappa}>0, \lambda_{\kappa}>0$ and $c_{\kappa}>0$ (which can depend only on $\kappa)$ such that for all $\lambda \in\left(0, \lambda_{\kappa}\right), \varepsilon \in\left(0, \varepsilon_{\kappa}\right)$ and for $\delta \in(0,1)$, there exists a unique solution $h \in \mathcal{C}_{\text {rad, }, \delta}^{2, \beta}\left(\mathbb{R}^{2}\right)$ of (18) such that

$$
v(x)=u_{1}(x)+h(x)
$$

solves (14) in $\bar{B}_{R_{\varepsilon, \lambda}}$. In addition

$$
\|h\|_{\mathcal{C}_{\text {rad, }, \delta}^{2, \beta}\left(\mathbb{R}^{2}\right)} \leq 2 c_{\kappa} r_{\varepsilon, \lambda}^{2} .
$$




\subsection{Analysis of the Laplace operator in weighted spaces}

In this section, we study the mapping properties of the Laplace operator in weighted Hölder spaces. Given $x_{1}, \ldots, x_{m} \in \Omega$, we define $\mathbf{x}:=\left(x_{1}, \ldots, x_{m}\right)$ and

$$
\bar{\Omega}^{*}(\mathbf{x}):=\bar{\Omega}-\left\{x_{1}, \ldots x_{m}\right\},
$$

and we choose $r_{0}>0$ so that the balls $B_{r_{0}}\left(x_{i}\right)$ of center $x_{i}$ and radius $r_{0}$ are mutually disjoint and included in $\Omega$. For all $r \in\left(0, r_{0}\right)$, we define

$$
\bar{\Omega}_{r}(\mathbf{x}):=\bar{\Omega}-\cup_{i=1}^{m} B_{r}\left(x_{i}\right) .
$$

Definition 4. Given $k \in \mathbb{R}, \beta \in(0,1)$ and $\nu \in \mathbb{R}$, we introduce the Hölder weighted space $\mathcal{C}_{\nu}^{k, \beta}\left(\bar{\Omega}^{*}(\mathbf{x})\right)$ as the space of functions $w \in \mathcal{C}_{\text {loc }}^{k, \beta}\left(\bar{\Omega}^{*}(\mathbf{x})\right)$ for which the following norm

$$
\|w\|_{\mathcal{C}_{\nu}^{k, \beta}\left(\bar{\Omega}^{*}(\mathbf{x})\right)}:=\|w\|_{\mathcal{C}^{k, \beta}\left(\bar{\Omega}_{r_{0} / 2}\right)}+\sum_{i=1}^{m} \sup _{0<r \leq r_{0 / 2}}\left(r^{-\nu}\left\|w\left(x_{i}+r \cdot\right)\right\|_{\mathcal{C}^{k, \beta}\left(\bar{B}_{2}-B_{1}\right)}\right)
$$

is finite.

When $k \geq 2$, we denote by $\left[\mathcal{C}_{\nu}^{k, \beta}\left(\bar{\Omega}^{*}(\mathbf{x})\right)\right]_{\mathbf{0}}$ to be the subspace of functions $w \in \mathcal{C}_{\nu}^{k, \beta}\left(\bar{\Omega}^{*}(\mathbf{x})\right)$ satisfying $w=0$ on $\partial \Omega$. We recall the following result.

Proposition 3. [2] Assume that $\nu<0$ and $\nu \notin \mathbb{Z}$, then

$$
\begin{aligned}
\mathcal{L}_{\nu}:\left[\mathcal{C}_{\nu}^{2, \beta}\left(\bar{\Omega}^{*}(\mathbf{x})\right)\right]_{\mathbf{0}} & \longrightarrow \mathcal{C}_{\nu-2}^{0, \beta}\left(\bar{\Omega}^{*}(\mathbf{x})\right) \\
w & \longmapsto \Delta w
\end{aligned}
$$

is surjective. Denote by $\tilde{\mathcal{G}}_{\nu}$ a right inverse of $\mathcal{L}_{\nu}$.

Remark 1. Observe that, when $\nu<0, \nu \notin \mathbb{Z}$, a right inverse is not unique and depends smoothly on the points $x_{1}, \ldots, x_{m}$, at least locally. Once a right inverse is fixed for one choice of the points $x_{1}, \ldots, x_{m}$, a right inverse for another choice of points $\tilde{x}_{1}, \ldots, \tilde{x}_{m}$ close to $x_{1}, \ldots, x_{m}$ can be obtained by using a simple perturbation argument.

\subsection{Harmonic extensions}

We study the properties of interior and exterior harmonic extensions. Given $\varphi \in \mathcal{C}^{2, \beta}\left(S^{1}\right)$, define $H^{i}\left(=H^{i}(\varphi ; \cdot)\right)$ to be the solution of

$$
\begin{cases}\Delta H^{i}=0 & \text { in } B_{1} \\ H^{i}=\varphi & \text { on } \partial B_{1} .\end{cases}
$$

We denote by $e_{1}, e_{2}$ the coordinate functions on $S^{1}$.

Lemma 2. [2] If we assume that

$$
\int_{S^{1}} \varphi d v_{S^{1}}=0 \quad \text { and } \quad \int_{S^{1}} \varphi e_{\ell} d v_{S^{1}}=0 \quad \text { for } \quad \ell=1,2
$$

then there exists $c>0$ such that

$$
\left\|H^{i}(\varphi ; \cdot)\right\|_{\mathcal{C}_{2}^{2, \beta}\left(\bar{B}_{1}^{*}\right)} \leq c\|\varphi\|_{\mathcal{C}^{2, \beta}\left(S^{1}\right)} .
$$


Given $\tilde{\varphi} \in \mathcal{C}^{2, \beta}\left(S^{1}\right)$ we define $H^{e}\left(=H^{e}(\tilde{\varphi} ; \cdot)\right)$ to be the solution of

$$
\begin{cases}\Delta H^{e}=0 & \text { in } \mathbb{R}^{2}-B_{1} \\ H^{e}=\tilde{\varphi} & \text { on } \partial B_{1}\end{cases}
$$

which decays at infinity.

Definition 5. Given $k \in \mathbb{N}, \beta \in(0,1)$ and $\nu \in \mathbb{R}$, we define the space $\mathcal{C}_{\nu}^{k, \beta}\left(\mathbb{R}^{2}-\right.$ $\left.B_{1}\right)$ as the space of functions $w \in \mathcal{C}_{l o c}^{k, \beta}\left(\mathbb{R}^{2}-B_{1}\right)$ for which the following norm

$$
\|w\|_{\mathcal{C}_{\nu}^{k, \beta}\left(\mathbb{R}^{2}-B_{1}\right)}=\sup _{r \geq 1}\left(r^{-\nu}\|w(r \cdot)\|_{\mathcal{C}_{\nu}^{k, \beta}\left(\bar{B}_{2}-B_{1}\right)}\right)
$$

is finite.

Lemma 3. [2] If we assume that

$$
\int_{S^{1}} \tilde{\varphi} d v_{S^{1}}=0,
$$

then there exists $c>0$ such that

$$
\left\|H^{e}(\tilde{\varphi}, ; \cdot)\right\|_{\mathcal{C}_{-1}^{2, \beta}\left(\mathbb{R}^{2}-B_{1}\right)} \leq c\|\tilde{\varphi}\|_{\mathcal{C}^{2, \beta}\left(S^{1}\right)} .
$$

If $F \subset L^{2}\left(S^{1}\right)$ is a space of functions defined on $S^{1}$, then we define the space $F^{\perp}$ to be the subspace of functions of $F$ which are $L^{2}\left(S^{1}\right)$-orthogonal to the functions $1, e_{1}$ and $e_{2}$. Then, we have:

Lemma 4. [2] The mapping

$$
\begin{aligned}
\mathcal{P}: \mathcal{C}^{2, \beta}\left(S^{1}\right)^{\perp} & \longrightarrow \mathcal{C}^{1, \beta}\left(S^{1}\right)^{\perp} \\
\psi & \longmapsto \partial_{r} H^{i}-\partial_{r} H^{e}
\end{aligned}
$$

where $H^{i}=H^{i}(\psi ; \cdot)$ and $H^{e}=H^{e}(\psi ; \cdot)$, is an isomorphism.

\section{The nonlinear interior problem}

We are interested in the study of equation

$$
\Delta w+\lambda|\nabla w|^{2}+2 e^{w}=0
$$

in $\bar{B}_{R_{\varepsilon, \lambda}}$.

Given $\varphi \in \mathcal{C}^{2, \beta}\left(S^{1}\right)$ satisfying $(22)$, recall that $u_{1}\left(=u_{\varepsilon=1, \tau=1}\right)$ and the solution $h$ of (18) satisfies (19). Define

$$
\mathbf{v}:=u_{1}+H^{i}\left(\varphi, \cdot / R_{\varepsilon, \lambda}\right)+h .
$$

Now, we look for a solution of (25) of the form $w=\mathbf{v}+v$. Using the fact that $H^{i}$ is harmonic, we see that this amounts to solve the equation

$$
\begin{aligned}
\mathbb{L} v= & \frac{8}{\left(1+r^{2}\right)^{2}} e^{h}\left(e^{H^{i}\left(\varphi, \cdot / R_{\varepsilon, \lambda}\right)+v}-v-1\right)+\frac{8}{\left(1+r^{2}\right)^{2}}\left(e^{h}-1\right) v \\
& +\lambda\left|\nabla\left[u_{1}+H^{i}\left(\varphi, \cdot / R_{\varepsilon, \lambda}\right)+h+v\right]\right|^{2}-\lambda\left|\nabla\left(u_{1}+h\right)\right|^{2} .
\end{aligned}
$$

We fix

$$
\mu \in(1,2)
$$


and denote by $\mathcal{G}_{\mu}$ to be a right inverse of $\mathbb{L}_{\mu}$ provided by Proposition 1 . To find a solution of (26) it is sufficient to find $v \in \mathcal{C}_{\mu}^{2, \beta}\left(\mathbb{R}^{2}\right)$ solution of

$$
v=\mathcal{G}_{\mu} \circ \mathcal{E}_{\mu} \circ \mathfrak{S}(v)
$$

where

$$
\begin{aligned}
\mathfrak{S}(v):= & \frac{8}{\left(1+r^{2}\right)^{2}} e^{h}\left(e^{H^{i}\left(\varphi, \cdot / R_{\varepsilon, \lambda}\right)+v}-v-1\right)+\frac{8}{\left(1+r^{2}\right)^{2}}\left(e^{h}-1\right) v \\
& +\lambda\left|\nabla\left[u_{1}+H^{i}\left(\varphi, \cdot / R_{\varepsilon, \lambda}\right)+h+v\right]\right|^{2}-\lambda\left|\nabla\left(u_{1}+h\right)\right|^{2} .
\end{aligned}
$$

We denote by $\mathcal{N}\left(=\mathcal{N}_{\varepsilon, \tau, \varphi}\right)$ the nonlinear operator appearing on the right-hand side of Eq. (27).

Given $\kappa>0$ (whose value will be fixed later on), we further assume that the functions $\varphi$ satisfy

$$
\|\varphi\|_{\mathcal{C}^{2, \beta}} \leq \kappa r_{\varepsilon, \lambda}^{2}
$$

Then, we have the following result.

Lemma 5. Given $\kappa>0$. There exist $\varepsilon_{\kappa}>0, \lambda_{\kappa}>0, c_{\kappa}>0$ and $\bar{c}_{\kappa}>0$ (which can depend only on $\kappa)$ such that for all $\lambda \in\left(0, \lambda_{\kappa}\right)$ and $\varepsilon \in\left(0, \varepsilon_{\kappa}\right)$

$$
\|\mathcal{N}(0)\|_{\mathcal{C}_{\mu}^{2, \beta}\left(\mathbb{R}^{2}\right)} \leq c_{\kappa} r_{\varepsilon, \lambda}^{2}
$$

and

$$
\left\|\mathcal{N}\left(v_{2}\right)-\mathcal{N}\left(v_{1}\right)\right\|_{\mathcal{C}_{\mu}^{2, \beta}\left(\mathbb{R}^{2}\right)} \leq \bar{c}_{\kappa} r_{\varepsilon, \lambda}^{2}\left\|v_{2}-v_{1}\right\|_{\mathcal{C}_{\mu}^{2, \beta}\left(\mathbb{R}^{2}\right)}
$$

provided that $v_{1}, v_{2} \in \mathcal{C}_{\mu}^{2, \beta}\left(\mathbb{R}^{2}\right)$ satisfy $\left\|v_{i}\right\|_{\mathcal{C}_{\mu}^{2, \beta}\left(\mathbb{R}^{2}\right)} \leq 2 c_{\kappa} r_{\varepsilon, \lambda}^{2}$.

Proof. The proof of the first estimate follows from the asymptotic behavior of $H^{i}$ together with the assumption on the norm of boundary data $\varphi$ given by (28). Indeed, let $c_{\kappa}$ be a constant depending only on $\kappa$ (provided $\varepsilon$ and $\lambda$ are chosen small enough), it follows from the estimate of $H^{i}$, given by Lemma 2 , that

$$
\left\|H^{i}\left(\cdot / R_{\varepsilon, \lambda}\right)\right\|_{\mathcal{C}_{2}^{2, \beta}\left(\bar{B}_{R_{\varepsilon, \lambda}}\right)} \leq c_{\kappa} R_{\varepsilon, \lambda}^{-2}\|\varphi\|_{\mathcal{C}^{2, \beta}\left(\bar{B}_{R_{\varepsilon, \lambda}}\right)} \leq c_{\kappa} \varepsilon^{2} .
$$

Since for each $x \in \bar{B}_{R_{\varepsilon, \lambda}}$, we have

$$
|h(x)| \leq c_{\kappa} r_{\varepsilon, \lambda}^{2+\delta} \varepsilon^{-\delta}= \begin{cases}\varepsilon^{1-\delta / 2} & \text { for } \varepsilon \geq \lambda \\ \lambda^{1+\delta / 2} \varepsilon^{-\delta} & \text { for } \lambda>\varepsilon\end{cases}
$$

then using condition $(A)$, we prove that $|h(x)| \longrightarrow 0$ as $\varepsilon$ and $\lambda$ tend to 0 . Given $\kappa>0$, there exists $c_{\kappa}>0$ such that

$$
\left\|\left(1+|\cdot|^{2}\right)^{-2} e^{h}\left(e^{H^{i}\left(\varphi ; \cdot / R_{\varepsilon, \lambda}\right)}-1\right)\right\|_{\mathcal{C}_{\mu-2}^{0, \beta}\left(\bar{B}_{R_{\varepsilon, \lambda}}\right)} \leq c_{\kappa} \varepsilon^{2} .
$$

On the other hand, using also condition $(A)$, we get

$$
\lambda \sup _{r \leq R_{\varepsilon, \lambda}} r^{2-\mu}\left|\nabla\left[u_{1}+H^{i}\left(\varphi, \cdot / R_{\varepsilon, \lambda}\right)+h\right]\right|^{2} \leq c_{\kappa} r_{\varepsilon, \lambda}^{2}
$$

and

$$
\lambda \sup _{r \leq R_{\varepsilon, \lambda}} r^{2-\mu}\left|\nabla\left[u_{1}+h\right]\right|^{2} \leq c_{\kappa} r_{\varepsilon, \lambda}^{2}
$$


Making use of Proposition 1 together with (17), we get for $\mu \in(1,2)$

$$
\|\mathcal{N}(0)\|_{\mathcal{C}_{\mu}^{2, \beta}\left(\mathbb{R}^{2}\right)} \leq c_{\kappa} r_{\varepsilon, \lambda}^{2}
$$

To derive the second estimate, we use the fact that, for $v_{1}, v_{2} \in \mathcal{C}_{\mu}^{2, \beta}\left(\mathbb{R}^{2}\right)$ satisfying $\left\|v_{i}\right\|_{\mathcal{C}_{\mu}^{2, \beta}\left(\mathbb{R}^{2}\right)} \leq 2 c_{\kappa} r_{\varepsilon, \lambda}^{2}$ for $i=1,2, \mu \in(1,2)$ and condition $(A)$. Hence there exists $c_{\kappa}>0$ such that

$$
\begin{aligned}
\sup _{r \leq R_{\varepsilon, \lambda}} & r^{2-\mu}\left|\mathfrak{S}\left(v_{2}\right)-\mathfrak{S}\left(v_{1}\right)\right| \\
\leq & c_{\kappa} r_{\varepsilon, \lambda}^{2}\left\|v_{2}-v_{1}\right\|_{\mathcal{C}_{\mu}^{2, \beta}\left(\mathbb{R}^{2}\right)}+c_{\kappa} \lambda\left\|v_{2}-v_{1}\right\|_{\mathcal{C}_{\mu}^{2, \beta}\left(\mathbb{R}^{2}\right)} \\
& +c_{\kappa} r_{\varepsilon, \lambda}^{2}\left\|v_{2}-v_{1}\right\|_{\mathcal{C}_{\mu}^{2, \beta}\left(\mathbb{R}^{2}\right)} .
\end{aligned}
$$

Similarly, making use of Proposition 1 together with (17), we conclude that there exists $\bar{c}_{\kappa}>0$ such that

$$
\left\|\mathcal{N}\left(v_{2}\right)-\mathcal{N}\left(v_{1}\right)\right\|_{\mathcal{C}_{\mu}^{2, \beta}\left(\bar{B}_{R_{\varepsilon, \lambda}}\right)} \leq \bar{c}_{\kappa} r_{\varepsilon, \lambda}^{2}\left\|v_{2}-v_{1}\right\|_{\mathcal{C}_{\mu}^{2, \beta}\left(\mathbb{R}^{2}\right)} .
$$

Reducing $\lambda_{\kappa}>0$ and $\varepsilon_{\kappa}>0$ if necessary, we can assume that,

$$
\bar{c}_{\kappa} r_{\varepsilon, \lambda}^{2} \leq \frac{1}{2}
$$

for all $\lambda \in\left(0, \lambda_{\kappa}\right)$ and $\varepsilon \in\left(0, \varepsilon_{\kappa}\right)$. Then, $(29)$ and (30) are enough to show that

$$
v \longmapsto \mathcal{N}(v)
$$

is a contraction from the ball

$$
\left\{v \in \mathcal{C}_{\mu}^{2, \beta}\left(\mathbb{R}^{2}\right):\|v\|_{\mathcal{C}_{\mu}^{2, \beta}\left(\mathbb{R}^{2}\right)} \leq 2 c_{\kappa} r_{\varepsilon, \lambda}^{2}\right\}
$$

into itself and hence has a unique fixed point $v\left(=\bar{v}_{\varepsilon, \tau, \varphi}\right)$ in this set. This fixed point is a solution of $(27)$ in $\mathbb{R}^{2}$. We summarize this in the following proposition.

Proposition 4. Given $\kappa>0$, there exist $\varepsilon_{\kappa}>0, \lambda_{\kappa}>0$ and $c_{\kappa}>0$ such that for all $\varepsilon \in\left(0, \varepsilon_{\kappa}\right), \lambda \in\left(0, \lambda_{\kappa}\right)$ satisfying $(A)$, for all $\tau$ in some fixed compact subset of $\left[\tau_{-}, \tau^{+}\right] \subset(0, \infty)$ and for a given $\varphi$ satisfying $(22)-(28)$, there exists a unique $v\left(:=\bar{v}_{\varepsilon, \tau, \varphi}\right)$ solution of $(27)$ such that

$$
w:=u_{1}+H^{i}\left(\varphi, \cdot / R_{\varepsilon, \lambda}\right)+h+\bar{v}_{\varepsilon, \tau, \varphi}
$$

solves $(25)$ in $\bar{B}_{R_{\varepsilon, \lambda}}$. In addition

$$
\|v\|_{\mathcal{C}_{\mu}^{2, \beta}\left(\mathbb{R}^{2}\right)} \leq 2 \bar{c}_{\kappa} r_{\varepsilon, \lambda}^{2}
$$

Observe that the function $v\left(:=\bar{v}_{\varepsilon, \tau, \varphi}\right)$ obtained as a fixed point for contraction mappings, depends continuously on the parameter $\tau$. 


\section{The nonlinear exterior problem}

Recall that $G(\cdot, \tilde{x})$ denotes the unique solution of

$$
-\Delta G(\cdot, \tilde{x})=8 \pi \delta_{\tilde{x}}
$$

in $\Omega$, with $G(\cdot, \tilde{x})=0$ on $\partial \Omega$. In addition the following decomposition holds

$$
G(x, \tilde{x})=-4 \log |x-\tilde{x}|+H(x, \tilde{x})
$$

where $x \longmapsto H(x, \tilde{x})$ is a smooth function. Here we will give an estimate of the gradient of $H(x, \tilde{x})$ without proof (see [22] and more details in [18], Lemma 2.1). There exists a constant $c>0$, so that

$$
\left|\nabla_{x} H(x, \tilde{x})\right| \leq c \log |x-\tilde{x}| .
$$

Let $\tilde{\mathbf{x}}:=\left(\tilde{x}_{1}, \ldots, \tilde{x}_{m}\right)$ close enough to $\mathbf{x}:=\left(x_{1}, \ldots, x_{m}\right), \tilde{\eta}:=$ $\left(\tilde{\eta}^{1}, \ldots, \tilde{\eta}^{m}\right) \in \mathbb{R}^{m}$ close to 0 and $\tilde{\varphi}:=\left(\tilde{\varphi}^{1}, \ldots, \tilde{\varphi}^{m}\right) \in\left(\mathcal{C}^{2, \beta}\left(S^{1}\right)\right)^{m}$ satisfying (24). We define

$$
\tilde{\mathbf{v}}:=\sum_{i=1}^{m}\left(1+\tilde{\eta}^{i}\right) G\left(\cdot, \tilde{x}_{i}\right)+\sum_{i=1}^{m} \chi_{r_{0}}\left(\cdot-\tilde{x}_{i}\right) H^{e}\left(\tilde{\varphi}^{i} ;\left(\cdot-\tilde{x}_{i}\right) / r_{\varepsilon, \lambda}\right)
$$

where $\chi_{r_{0}}$ is a cutoff function identically equal to 1 in $B_{r_{0} / 2}$ and identically equal to 0 outside $B_{r_{0}}$. We would like to find a solution of the equation

$$
\Delta u+\lambda|\nabla u|^{2}+\rho^{2} e^{u}=0
$$

which is defined in $\bar{\Omega}_{r_{\varepsilon, \lambda}}(\tilde{\mathbf{x}}):=\bar{\Omega}-\cup_{i=1}^{m} B_{r_{\varepsilon, \lambda}}\left(\tilde{x}_{i}\right)$ and is a perturbation of $\tilde{\mathbf{v}}$. Writing $v=\tilde{\mathbf{v}}+\tilde{v}$, this amounts to solve

$$
-\Delta \tilde{v}=\rho^{2} e^{\tilde{\mathbf{v}}+\tilde{v}}+\lambda|\nabla(\tilde{\mathbf{v}}+\tilde{v})|^{2}+\Delta \tilde{\mathbf{v}} .
$$

We need to define an auxiliary weighted space:

Definition 6. Let $\bar{r} \in\left(0, r_{0} / 2\right), k \in \mathbb{R}, \beta \in(0,1)$ and $\nu \in \mathbb{R}$, we define the Hölder weighted space $\mathcal{C}_{\nu}^{k, \beta}\left(\bar{\Omega}_{\bar{r}}(\mathbf{x})\right)$ as the set of functions $w \in \mathcal{C}^{k, \beta}\left(\bar{\Omega}_{\bar{r}}(\mathbf{x})\right)$ for which the following norm

$$
\begin{aligned}
\|w\|_{\mathcal{C}_{\nu}^{k, \beta}\left(\bar{\Omega}_{\bar{r}}(\mathbf{x})\right)}:= & \|w\|_{\mathcal{C}^{k, \beta}\left(\bar{\Omega}_{r_{0} / 2}(\mathbf{x})\right)} \\
& +\sum_{i=1}^{m} \sup _{r \in\left[\bar{r}, r_{0} / 2\right)}\left(r^{-\nu}\left\|w\left(x_{i}+r \cdot\right)\right\|_{\mathcal{C}^{k, \beta}\left(\bar{B}_{2}-B_{1}\right)}\right) .
\end{aligned}
$$

is finite

For all $\sigma \in\left(0, r_{0} / 2\right)$ and all $Y=\left(y_{1}, \ldots, y_{m}\right) \in \Omega^{m}$ such that $\|X-Y\| \leq r_{0} / 2$, where $X=\left(x_{1}, \ldots, x_{m}\right)$, we denote by

$$
\tilde{\mathcal{E}}_{\sigma, Y}: \mathcal{C}_{\nu}^{0, \beta}\left(\bar{\Omega}_{\sigma}(Y)\right) \longrightarrow \mathcal{C}_{\nu}^{0, \beta}\left(\bar{\Omega}^{*}(Y)\right)
$$

the extension operator defined by $\tilde{\mathcal{E}}_{\sigma, Y}(f)=f$ in $\bar{\Omega}_{\sigma}(Y)$

$$
\tilde{\mathcal{E}}_{\sigma, Y}(f)\left(y_{i}+x\right)=\tilde{\chi}\left(\frac{|x|}{\sigma}\right) f\left(y_{i}+\sigma \frac{x}{|x|}\right)
$$


in $B_{\sigma}\left(y_{i}\right)-B_{\sigma / 2}\left(y_{i}\right)$, for each $i=1, \ldots, m$ and $\tilde{\mathcal{E}}_{\sigma, Y}(f)=0$ in each $B_{\sigma / 2}\left(y_{i}\right)$, where $t \longmapsto \tilde{\chi}(t)$ is a cutoff function identically equal to 1 for $t \geq 1$ and identically equal to 0 for $t \leq 1 / 2$. It is easy to check that there exists a constant $c=c(\nu)>0$ only depending on $\nu$ such that

$$
\left\|\tilde{\mathcal{E}}_{\sigma, Y}(w)\right\|_{\mathcal{C}_{\nu}^{0, \beta}\left(\bar{\Omega}^{*}(Y)\right)} \leq c\|w\|_{\mathcal{C}_{\nu}^{0, \beta}\left(\bar{\Omega}_{\sigma}(Y)\right)} .
$$

We fix

$$
\nu \in(-1,0),
$$

and denote by $\tilde{\mathcal{G}}_{\nu}: \mathcal{C}_{\nu-2}^{0, \beta}\left(\bar{\Omega}^{*}(\tilde{\mathbf{x}})\right) \longrightarrow \mathcal{C}_{\nu}^{2, \beta}\left(\bar{\Omega}^{*}(\tilde{\mathbf{x}})\right)$ a right inverse of $\Delta$ provided by Proposition 3 with $\bar{\Omega}^{*}(\tilde{\mathbf{x}})=\bar{\Omega}-\left\{\tilde{x}_{1}, \ldots, \tilde{x}_{m}\right\}$. Clearly, it is enough to find $\tilde{v} \in \mathcal{C}_{\nu}^{2, \beta}\left(\bar{\Omega}^{*}(\tilde{\mathbf{x}})\right)$ solution of

$$
\tilde{v}=\tilde{\mathcal{G}}_{\nu} \circ \tilde{\mathcal{E}}_{r_{\varepsilon, \lambda}, \tilde{\mathbf{x}}}\left(\rho^{2} e^{\tilde{\mathbf{v}}+\tilde{v}}+\lambda|\nabla(\tilde{\mathbf{v}}+\tilde{v})|^{2}+\Delta \tilde{\mathbf{v}}\right)=\tilde{\mathcal{G}}_{\nu} \circ \tilde{\mathcal{E}}_{r_{\varepsilon, \lambda}, \tilde{\mathbf{x}}} \circ \tilde{\mathfrak{R}}(\tilde{v}),
$$

where

$$
\tilde{\mathfrak{R}}(\tilde{v})=\rho^{2} e^{\tilde{\mathbf{v}}+\tilde{v}}+\lambda|\nabla(\tilde{\mathbf{v}}+\tilde{v})|^{2}+\Delta \tilde{\mathbf{v}} .
$$

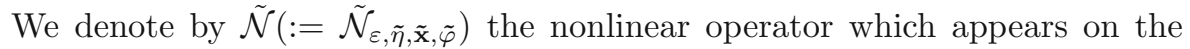
right-hand side of Eq. (34).

Given $\kappa>0$, we assume that the point $\tilde{\mathbf{x}}=\left(\tilde{x}_{1}, \ldots, \tilde{x}_{m}\right)$, the function $\tilde{\varphi}=\left(\varphi_{1}, \ldots, \varphi_{m}\right)$ and the parameter $\tilde{\eta}=\left(\tilde{\eta}^{1}, \ldots, \tilde{\eta}^{m}\right)$ satisfy

$$
\begin{aligned}
\left|\tilde{x}_{i}-x_{i}\right| & \leq \kappa r_{\varepsilon, \lambda}, \\
\left\|\tilde{\varphi}^{i}\right\|_{\mathcal{C}^{2, \beta}} & \leq \kappa r_{\varepsilon, \lambda}^{2}
\end{aligned}
$$

and

$$
\left|\tilde{\eta}^{i}\right| \leq \kappa r_{\varepsilon, \lambda}^{2}
$$

Then, the following result holds.

Lemma 6. Given $\kappa>0$, there exist $\varepsilon_{\kappa}>0, \lambda_{\kappa}>0, c_{\kappa}>0$ and $\bar{c}_{\kappa}>0$ such that for all $\varepsilon \in\left(0, \varepsilon_{\kappa}\right), \lambda \in\left(0, \lambda_{\kappa}\right)$

$$
\|\tilde{\mathcal{N}}(0)\|_{\mathcal{C}_{\nu}^{2, \beta}\left(\bar{\Omega}^{*}(\tilde{\mathbf{x}})\right)} \leq c_{\kappa} r_{\varepsilon, \lambda}^{2}
$$

and

$$
\left\|\tilde{\mathcal{N}}\left(\tilde{v}_{2}\right)-\tilde{\mathcal{N}}\left(\tilde{v}_{1}\right)\right\|_{\mathcal{C}_{\nu}^{2, \beta}\left(\bar{\Omega}^{*}(\tilde{\mathbf{x}})\right)} \leq \bar{c}_{\kappa} r_{\varepsilon, \lambda}^{2}\left\|\tilde{v}_{2}-\tilde{v}_{1}\right\|_{\mathcal{C}_{\nu}^{2, \beta}\left(\bar{\Omega}^{*}(\tilde{\mathbf{x}})\right)}
$$

provided $\tilde{v}_{1}, \tilde{v}_{2} \in \mathcal{C}_{\nu}^{2, \beta}\left(\bar{\Omega}^{*}(\tilde{\mathbf{x}})\right)$ and satisfying $\left\|\tilde{v}_{i}\right\|_{\mathcal{C}_{\nu}^{2, \beta}\left(\bar{\Omega}^{*}(\tilde{\mathbf{x}})\right)} \leq 2 c_{\kappa} r_{\varepsilon, \lambda}^{2}$.

Proof. The proof of the first estimate follows from the asymptotic behavior of $H^{e}$ together with the assumption on the norm of boundary data $\tilde{\varphi}^{i}$ given by (36). Indeed, let $c_{\kappa}$ be a constant depending only on $\kappa$ (provided $\varepsilon$ and $\lambda$ are chosen small enough) it follows from the estimate of $H^{e}$, given by Lemma 3 , that

$$
\left|H^{e}\left(\tilde{\varphi}^{i} ;\left(x-\tilde{x}_{i}\right) / r_{\varepsilon, \lambda}\right)\right| \leq c_{\kappa} r_{\varepsilon, \lambda}^{3} r^{-1} .
$$

Recall that $\tilde{\mathcal{N}}(\tilde{v})=\tilde{\mathcal{G}}_{\nu} \circ \tilde{\mathfrak{R}}(\tilde{v})$, we will estimate $\tilde{\mathcal{N}}(0)$ in different subregions of $\bar{\Omega}^{*}(\tilde{\mathbf{x}})$. 
- In $B_{r_{0}}\left(\tilde{x}_{i}\right)$, we have $\chi_{r_{0}}\left(x-\tilde{x}_{i}\right)=1$ and $\Delta \tilde{\mathbf{v}}=0$ so that

$$
\begin{aligned}
|\tilde{\mathfrak{R}}(0)| \leq & c_{\kappa} \varepsilon^{2}\left|x-\tilde{x}_{i}\right|^{-4\left(1+\tilde{\eta}^{i}\right)} \prod_{\ell=1, \ell \neq i}^{m}\left|x-\tilde{x}_{\ell}\right|^{-4\left(1+\tilde{\eta}^{\ell}\right)}+\lambda|\nabla \tilde{\mathbf{v}}|^{2} \\
\leq & c_{\kappa} \varepsilon^{2} r^{-4\left(1+\tilde{\eta}^{i}\right)}+\lambda\left|4\left(1+\tilde{\eta}^{i}\right) r^{-1}+\left(1+\tilde{\eta}^{i}\right)\right| \nabla_{x} H(x, \tilde{x}) \mid \\
& +\left|\nabla H^{e}\left(\tilde{\varphi}^{i} ;\left(\cdot-\tilde{x}_{i}\right) / r_{\varepsilon, \lambda}\right)\right|^{2} \\
\leq & c_{\kappa} \varepsilon^{2} r^{-4\left(1+\tilde{\eta}^{i}\right)}+c_{\kappa} \lambda\left(\left(1+\tilde{\eta}^{i}\right) r^{-1}+\left(1+\tilde{\eta}^{i}\right)|\log r|+r_{\varepsilon, \lambda}^{2} r^{-2}\right)^{2} .
\end{aligned}
$$

Hence, for $\nu \in(-1,0)$ and $\tilde{\eta}^{i}$ small enough, we get

$$
\|\tilde{\Re}(0)\|_{\mathcal{C}_{\nu-2}^{0, \beta}\left(\bigcup_{i=1}^{m} B_{r_{0}}\left(\tilde{x}_{i}\right)\right)} \leq \sup _{r_{\varepsilon, \lambda} \leq r \leq r_{0} / 2} r^{2-\nu}|\tilde{\Re}(0)| \leq c_{\kappa} \varepsilon^{2} r_{\varepsilon, \lambda}^{-2}+c_{\kappa} \lambda .
$$

- In $\bar{\Omega}-B_{r_{0}}\left(\tilde{x}_{i}\right)$, we have $\chi_{r_{0}}\left(x-\tilde{x}_{i}\right)=0$ and $\Delta \tilde{\mathbf{v}}=0$. Thus

$$
|\tilde{\Re}(0)| \leq c_{\kappa} \varepsilon^{2} \prod_{\ell=1}^{m} e^{\left(1+\tilde{\eta}^{\ell}\right) G\left(x, \tilde{x}_{\ell}\right)}+c_{\kappa} \lambda\left(\left(1+\tilde{\eta}^{i}\right) r^{-1}+\left(1+\tilde{\eta}^{i}\right)|\log r|+r_{\varepsilon, \lambda}^{2} r^{-2}\right)^{2} .
$$

So, for $\nu \in(-1,0)$, we have

$$
\|\tilde{\Re}(0)\|_{\mathcal{C}_{\nu-2}^{0, \beta}\left(\bar{\Omega}-\bigcup_{i=1}^{m} B_{r_{0}}\left(\tilde{x}_{i}\right)\right)} \leq \sup _{r_{0} \leq r} r^{2-\nu}|\tilde{\mathfrak{R}}(0)| \leq c_{\kappa} \varepsilon^{2}+c_{\kappa} \lambda .
$$

- In $B_{r_{0}}\left(\tilde{x}_{i}\right)-B_{r_{0} / 2}\left(\tilde{x}_{i}\right)$, using the estimate (38), then we have

$$
\begin{aligned}
|\tilde{\mathfrak{R}}(0)| \leq & c_{\kappa} \varepsilon^{2} r^{-4\left(1+\tilde{\eta}^{j_{i}}\right)}+c_{\kappa} \lambda\left(\left(1+\tilde{\eta}^{i}\right) r^{-1}+\left(1+\tilde{\eta}^{i}\right)|\log r|+r_{\varepsilon, \lambda}^{2} r^{-2}\right)^{2} \\
& +\sum_{i=1}^{m}\left|\left[\Delta, \chi_{r_{0}}\left(x-\tilde{x}_{i}\right)\right]\right|\left|H^{e}\left(\tilde{\varphi}^{i} ;\left(x-\tilde{x}_{i}\right) / r_{\varepsilon, \lambda}\right)\right| \\
\leq & c_{\kappa}\left(\varepsilon^{2}+\lambda\left(\left(1+\tilde{\eta}^{i}\right) r^{-1}+\left(1+\tilde{\eta}^{i}\right)|\log r|+r_{\varepsilon, \lambda}^{2} r^{-2}\right)^{2}+r^{-1} r_{\varepsilon, \lambda}^{3}\right),
\end{aligned}
$$

where

$$
\left[\Delta, \chi_{r_{0}}\right] w=\Delta w \chi_{r_{0}}+w \Delta \chi_{r_{0}}+2 \nabla w \cdot \nabla \chi_{r_{0}}
$$

Then,

$$
\|\tilde{\mathfrak{R}}(0)\|_{\mathcal{C}_{\nu-2}^{0, \beta}\left(\left(B_{r_{0}}\left(\tilde{x}_{i}\right)-\bigcup_{i=1}^{m} B_{r_{0} / 2}\left(\tilde{x}_{i}\right)\right)\right)} \leq \sup _{r_{0} / 2 \leq r \leq r_{0}} r^{2-\nu}|\tilde{\mathfrak{R}}(0)| \leq c_{\kappa} r_{\varepsilon, \lambda}^{2}+c_{\kappa} \lambda
$$

So,

$$
\|\tilde{\Re}(0)\|_{\mathcal{C}_{\nu-2}^{0, \beta}\left(\left(\Omega-\bigcup_{i=1}^{m} B_{r_{0}}\left(\tilde{x}_{i}\right)\right)\right)} \leq c_{\kappa} r_{\varepsilon, \lambda}^{2} .
$$

Making use of Proposition 3 together with (33) we conclude that

$$
\|\tilde{\mathcal{N}}(0)\|_{\mathcal{C}_{\nu}^{2, \beta}\left(\bar{\Omega}^{*}(\mathbf{x})\right)} \leq c_{\kappa} r_{\varepsilon, \lambda}^{2} .
$$

For the proof of the second estimate, let $\tilde{v}_{1}$ and $\tilde{v}_{2} \in C_{\nu}^{2, \beta}\left(\bar{\Omega}^{*}(\tilde{\mathbf{x}})\right)$ satisfying $\left\|\tilde{v}_{i}\right\|_{\mathcal{C}_{\mu}^{2, \beta}\left(\bar{\Omega}^{*}(\tilde{\mathbf{x}})\right)} \leq 2 c_{\kappa} r_{\varepsilon, \lambda}^{2}$ for $i=1,2$, we have

$$
\left|\tilde{\mathfrak{R}}\left(\tilde{v}_{2}\right)-\tilde{\mathfrak{R}}\left(\tilde{v}_{1}\right)\right| \leq c_{\kappa} \varepsilon^{2} e^{\tilde{\mathbf{v}}}\left(e^{\tilde{v}_{2}}-e^{\tilde{v}_{1}}\right)|+\lambda|\left|\nabla\left(\tilde{\mathbf{v}}+\tilde{v}_{2}\right)\right|^{2}-\left|\nabla\left(\tilde{\mathbf{v}}+\tilde{v}_{1}\right)\right|^{2} \mid
$$


Then

$$
\begin{aligned}
\left|\tilde{\mathfrak{R}}\left(\tilde{v}_{2}\right)-\tilde{\mathfrak{R}}\left(\tilde{v}_{1}\right)\right| \leq & c_{\kappa} \varepsilon^{2}\left|x-x_{i}\right|^{-4\left(1+\tilde{\eta}^{i}\right)}\left|\tilde{v}_{2}-\tilde{v}_{1}\right| \\
& +c_{\kappa} \lambda\left|\nabla\left(\tilde{v}_{2}-\tilde{v}_{1}\right)\right|\left(\left|\nabla\left(\tilde{v}_{2}+\tilde{v}_{1}\right)\right|+2|\nabla \tilde{\mathbf{v}}|\right) \\
\leq & c_{\kappa} \varepsilon^{2} r^{-4\left(1+\tilde{\eta}^{j}\right)}\left|\tilde{v}_{2}-\tilde{v}_{1}\right| \\
& +c_{\kappa} \lambda\left|\nabla\left(\tilde{v}_{2}-\tilde{v}_{1}\right)\right|\left(\left|\nabla\left(\tilde{v}_{2}+\tilde{v}_{1}\right)\right|+2|\nabla \tilde{\mathbf{v}}|\right) .
\end{aligned}
$$

So, for $\tilde{\eta}^{i}$ small enough and using the estimate (33), there exists $\bar{c}_{\kappa}$ (depending on $\kappa$ ) such that

$$
\left\|\tilde{\mathcal{N}}\left(\tilde{v}_{2}\right)-\tilde{\mathcal{N}}\left(\tilde{v}_{1}\right)\right\|_{\mathcal{C}_{\nu}^{2, \beta}\left(\bar{\Omega}^{*}(\tilde{\mathbf{x}})\right)} \leq \bar{c}_{\kappa} r_{\varepsilon, \lambda}^{2}\left\|\tilde{v}_{2}-\tilde{v}_{1}\right\|_{\mathcal{C}_{\nu}^{2, \beta}\left(\bar{\Omega}^{*}(\tilde{\mathbf{x}})\right)} .
$$

Then we get the second estimate.

Reducing $\lambda_{\kappa}>0$ and $\varepsilon_{\kappa}>0$ if necessary, we can assume that

$$
\bar{c}_{\kappa} r_{\varepsilon, \lambda}^{2} \leq \frac{1}{2}
$$

for all $\lambda \in\left(0, \lambda_{\kappa}\right)$ and $\varepsilon \in\left(0, \varepsilon_{\kappa}\right)$. Then, (40) and (41) are enough to show that

$$
\tilde{v} \longmapsto \tilde{\mathcal{N}}(\tilde{v})
$$

is a contraction from the ball

$$
\left\{\tilde{v} \in \mathcal{C}_{\nu}^{2, \beta}\left(\mathbb{R}^{2}\right):\|\tilde{v}\|_{\mathcal{C}_{\nu}^{2, \beta}\left(\mathbb{R}^{2}\right)} \leq 2 c_{\kappa} r_{\varepsilon, \lambda}^{2}\right\}
$$

into itself and hence has a unique fixed point $\tilde{v}\left(=\bar{v}_{\varepsilon, \tau, \varphi}\right)$ in this set. This fixed point is a solution of (34). We summarize this in the following proposition

Proposition 5. Given $\kappa>0$, there exist $\varepsilon_{\kappa}>0, \lambda_{\kappa}>0$ and $c_{\kappa}>0$ (depending on $\kappa)$ such that for all $\varepsilon \in\left(0, \varepsilon_{\kappa}\right)$ and $\lambda \in\left(0, \lambda_{\kappa}\right)$, for all set of parameters $\tilde{\eta}^{i}$ satisfying (37), the points $\tilde{x}_{i}$ satisfying (35) and function $\tilde{\varphi}$ satisfying (24)-(36), there exists a unique $\tilde{v}\left(:=\tilde{v}_{\varepsilon, \tilde{\eta}, \tilde{\mathbf{x}}, \tilde{\varphi})}\right.$ solution of $(34)$ such that

$$
\tilde{u}:=\sum_{i=1}^{m}\left(1+\tilde{\eta}^{i}\right) G\left(\cdot, \tilde{x}_{i}\right)+\sum_{i=1}^{m} \chi_{r_{0}}\left(\cdot-\tilde{x}_{i}\right) H^{e}\left(\tilde{\varphi}^{i} ;\left(\cdot-\tilde{x}_{i}\right) / r_{\varepsilon, \lambda}\right)+\tilde{v}_{\varepsilon, \tilde{\eta}, \tilde{\mathbf{x}}, \tilde{\varphi}}
$$

solves $(32)$ in $\bar{\Omega}_{r_{\varepsilon, \lambda}}(\tilde{\mathbf{x}})$. In addition

$$
\|\tilde{v}\|_{\mathcal{C}_{\nu}^{2, \beta}\left(\bar{\Omega}^{*}(\mathbf{x})\right)} \leq 2 c_{\kappa} r_{\varepsilon, \lambda}^{2} .
$$

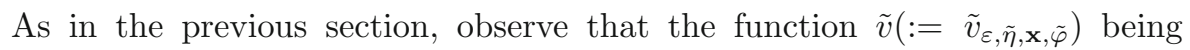
obtained as a fixed point for contraction mapping, depends smoothly on the parameters $\tilde{\eta}$ and the points $\tilde{\mathbf{x}}$.

\section{The nonlinear Cauchy-data matching}

Keeping the notations of the previous sections, we gather the results of Propositions 4 and 5 . Assume that $\tilde{\mathbf{x}}:=\left(\tilde{x}_{1}, \ldots, \tilde{x}_{m}\right) \in \Omega^{m}$ is given close to $\mathbf{x}:=\left(x_{1}, \ldots, x_{m}\right)$ and satisfies $(35)$. Assume also that $\tau:=\left(\tau_{1}, \ldots, \tau_{m}\right) \in$ $\left[\tau_{-}, \tau^{+}\right]^{m} \subset(0, \infty)^{m}$ is given (the values of $\tau_{-}$and $\tau^{+}$will be fixed shortly). 
First, we consider some set of boundary data $\varphi:=\left(\varphi^{1}, \ldots, \varphi^{m}\right) \in\left(\mathcal{C}^{2, \beta}\left(S^{1}\right)\right)^{m}$ satisfying (22). We set

$$
R_{\varepsilon, \lambda}^{i}=\tau_{i} r_{\varepsilon, \lambda} / \varepsilon
$$

and recall that

$$
\rho^{2}=\frac{8 \varepsilon^{2}}{\left(1+\varepsilon^{2}\right)^{2}}
$$

According to the result of Proposition 4, we can find $v_{i n t}^{i}$ a solution of

$$
\Delta u+\lambda|\nabla u|^{2}+\rho^{2} e^{u}=0
$$

in each $B_{r_{\varepsilon, \lambda}}\left(\tilde{x}_{i}\right)$ that can be decomposed as

$$
\begin{aligned}
v_{i n t}^{i}(x)= & v_{\varepsilon, \tau_{i}}\left(x-\tilde{x}_{i}\right)+h\left(R_{\varepsilon, \lambda}^{i}\left(x-\tilde{x}_{i}\right) / r_{\varepsilon, \lambda}\right)+H^{i}\left(\varphi^{i} ;\left(x-\tilde{x}_{i}\right) / r_{\varepsilon, \lambda}\right) \\
& +\bar{v}_{\varepsilon, \tau_{i}, \varphi^{i}}\left(R_{\varepsilon, \lambda}^{i}\left(x-\tilde{x}_{i}\right) / r_{\varepsilon, \lambda}\right),
\end{aligned}
$$

where the function $v^{i}=\bar{v}_{\varepsilon, \tau_{i}, \varphi^{i}}$ satisfies

$$
\left\|v^{i}\right\|_{\mathcal{C}_{\mu}^{2, \beta}\left(\mathbb{R}^{2}\right)} \leq 2 c_{\kappa} r_{\varepsilon, \lambda}^{2} .
$$

Similarly, given some boundary data $\tilde{\varphi}=\left(\tilde{\varphi}^{1}, \ldots, \tilde{\varphi}^{m}\right) \in\left(\mathcal{C}^{2, \beta}\left(S^{1}\right)\right)^{m}$ satisfying (24), some parameters $\tilde{\eta}:=\left(\tilde{\eta}^{1}, \ldots, \tilde{\eta}^{m}\right) \in \mathbb{R}^{m}$ satisfying (37), provided $\varepsilon \in\left(0, \varepsilon_{\kappa}\right)$ and $\lambda \in\left(0, \lambda_{\kappa}\right)$, we use the result of Proposition 5 , to find a solution $v_{\text {ext }}$ of (42) which can be decomposed as

$$
v_{\text {ext }}=\sum_{i=1}^{m}\left(1+\tilde{\eta}^{i}\right) G\left(\cdot, \tilde{x}_{i}\right)+\sum_{i=1}^{m} \chi_{r_{0}}\left(\cdot-\tilde{x}_{i}\right) H^{e}\left(\tilde{\varphi}^{i} ;\left(\cdot-\tilde{x}_{i}\right) / r_{\varepsilon, \lambda}\right)+\tilde{v}_{\varepsilon, \tilde{\eta}, \tilde{\mathbf{x}}, \tilde{\varphi}}
$$

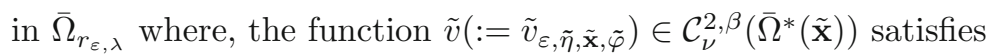

$$
\|\tilde{v}\|_{\mathcal{C}_{\nu}^{2, \beta}\left(\bar{\Omega}^{*}(\tilde{\mathbf{x}})\right)} \leq 2 c_{\kappa} r_{\varepsilon, \lambda}^{2} .
$$

It remains to determine the parameters and the functions in such a way that the function which is equal to $v_{i n t}^{i}$ in $\cup_{i=1}^{m} B_{r_{\varepsilon, \lambda}}\left(\tilde{x}_{i}\right)$ and that is equal to $v_{\text {ext }}$ in $\left.\bar{\Omega}_{r_{\varepsilon, \lambda}}(\tilde{\mathbf{x}})\right)$ is smooth. This amounts to find the boundary data and the parameters so that, for each $i=1, \ldots, m$

$$
v_{\text {int }}^{i}=v_{\text {ext }} \quad \text { and } \quad \partial_{r} v_{\text {int }}^{i}=\partial_{r} v_{\text {ext }},
$$

on $\partial B_{r_{\varepsilon, \lambda}}\left(\tilde{x}_{i}\right)$. Assuming we have already done so, this provides for each $\varepsilon$ and $\lambda$ small enough a function $v_{\varepsilon, \lambda} \in \mathcal{C}^{2, \beta}$ (which is obtained by patching together the functions $v_{\text {int }}^{i}$ and the function $v_{\text {ext }}$ ) solution of $-\Delta u-\lambda|\nabla u|^{2}=\rho^{2} e^{u}$ and the elliptic regularity theory implies that this solution is in fact smooth. This will complete the proof of our result since, as $\varepsilon$ and $\lambda$ tend to 0 , the sequence of solutions we have obtained satisfies the required properties, namely, away from the points $x_{i}$ the sequence $v_{\varepsilon, \lambda}$ converges to $\sum_{i} G\left(\cdot, x_{i}\right)$.

Before we proceed, the following remarks are due. First, it will be convenient to observe that the function $v_{\varepsilon, \tau_{i}}$ can be expanded as

$$
v_{\varepsilon, \tau_{i}}(x)=-2 \log \tau_{i}-4 \log |x|+\mathcal{O}\left(\frac{\varepsilon^{2} \tau_{i}^{-2}}{|x|^{2}}\right)
$$


near $\partial B_{r_{\varepsilon, \lambda}}$. The function

$$
\sum_{\ell=1}^{m}\left(1+\tilde{\eta}^{\ell}\right) G\left(x, \tilde{x}_{\ell}\right)
$$

which appear in the expression of $v_{\text {ext }}$ can be expanded as

$$
\begin{aligned}
\sum_{\ell=1}^{m}\left(1+\tilde{\eta}^{\ell}\right) G\left(x+\tilde{x}_{i}, \tilde{x}_{\ell}\right)= & -4\left(1+\tilde{\eta}^{i}\right) \log |x|+\mathcal{F}_{i}\left(\tilde{\mathbf{x}} ; \tilde{x}_{i}\right) \\
& +\nabla \mathcal{F}_{i}\left(\tilde{\mathbf{x}} ; \tilde{x}_{i}\right) \cdot x+\mathcal{O}\left(r_{\varepsilon, \lambda}^{2}\right)
\end{aligned}
$$

near $\partial B_{r_{\varepsilon, \lambda}}\left(\tilde{x}_{i}\right)$. Here, we have defined

$$
\mathcal{F}_{i}(\tilde{\mathbf{x}} ; \cdot):=H\left(\tilde{x}_{i}, \cdot\right)+\sum_{\ell \neq i} G\left(\tilde{x}_{\ell}, \cdot\right) .
$$

Thus for $x$ near $\partial B_{r_{\varepsilon, \lambda}}$, we have

$$
\begin{aligned}
\left(v_{i n t}^{i}\right. & \left.-v_{\text {ext }}\right)(x) \\
= & -2 \log \tau_{i}+4 \tilde{\eta}^{i} \log \left|x-\tilde{x}_{i}\right|+h\left(R_{\varepsilon, \lambda}^{i}\left(x-\tilde{x}_{i}\right) / r_{\varepsilon, \lambda}\right) \\
& +H^{i}\left(\varphi^{i} ;\left(x-\tilde{x}_{i}\right) / r_{\varepsilon, \lambda}\right)-H^{e}\left(\tilde{\varphi}^{i} ;\left(x-\tilde{x}_{i}\right) / r_{\varepsilon, \lambda}\right) \\
& -\left(\left(1+\tilde{\eta}^{i}\right) H\left(x, \tilde{x}_{i}\right)+\sum_{\ell=1, \ell \neq i}^{m}\left(1+\tilde{\eta}^{\ell}\right) G\left(x, \tilde{x}_{\ell}\right)\right) \\
& +\mathcal{O}\left(\frac{\varepsilon^{2} \tau_{i}^{-2}}{\left|x-\tilde{x}_{i}\right|^{2}}\right)+\mathcal{O}\left(r_{\varepsilon, \lambda}^{2}\right) \\
= & -2 \log \tau_{i}+4 \tilde{\eta}^{i} \log |x|-\left(\left(1+\tilde{\eta}^{i}\right) H\left(\tilde{x}_{i}, \tilde{x}_{i}\right)+\sum_{\ell=1, \ell \neq i}^{m}\left(1+\tilde{\eta}^{\ell}\right) G\left(\tilde{x}_{i}, \tilde{x}_{\ell}\right)\right) \\
& +\mathcal{O}\left(\left|x-\tilde{x}_{i}\right|^{2}\right)+\mathcal{O}\left(\frac{\varepsilon^{2} \tau_{i}^{-2}}{\left|x-\tilde{x}_{i}\right|^{2}}\right)+\mathcal{O}\left(r_{\varepsilon, \lambda}^{2}\right) \\
= & -2 \log \tau_{i}+4 \tilde{\eta}^{i} \log r_{\varepsilon, \lambda}-\mathcal{F}_{i}\left(\tilde{x}_{i}, \tilde{\mathbf{x}}\right)+\mathcal{O}(\varepsilon)+\mathcal{O}\left(r_{\varepsilon, \lambda}^{2}\right) \\
= & -2 \log \tau_{i}+4 \tilde{\eta}^{i} \log r_{\varepsilon, \lambda}-\mathcal{F}_{i}\left(\tilde{x}_{i}, \tilde{\mathbf{x}}\right)+\mathcal{O}\left(r_{\varepsilon, \lambda}^{2}\right)
\end{aligned}
$$

where $\tilde{\mathbf{x}}=\left(\tilde{x}_{1}, \ldots, \tilde{x}_{m}\right)$.

Next, in (45), all functions are defined on $\partial B_{r_{\varepsilon, \lambda}}\left(\tilde{x}_{i}\right)$, but it will be convenient to solve the following equations

$$
\left(v_{i n t}^{i}-v_{e x t}\right)\left(\tilde{x}_{i}+r_{\varepsilon, \lambda} \cdot\right)=0 \quad \text { and } \quad \partial_{r}\left(\left(v_{i n t}^{i}-v_{e x t}\right)\left(\tilde{x}_{i}+r_{\varepsilon, \lambda} \cdot\right)\right)=0
$$

on $S^{1}$. Here all functions are considered as functions of $y \in S^{1}$ and we have simply used the change of variables $x=\tilde{x}_{i}+r_{\varepsilon, \lambda} y$ to parameterize $\partial B_{r_{\varepsilon, \lambda}}\left(\tilde{x}_{i}\right)$.

Since the boundary data we have chosen satisfy (22) and (24), we can decompose

$$
\varphi^{i}=\varphi_{0}^{i}+\varphi_{1}^{i}+\varphi^{i, \perp} \quad \text { and } \quad \tilde{\varphi}^{i}=\tilde{\varphi}_{0}^{i}+\tilde{\varphi}_{1}^{i}+\tilde{\varphi}^{i, \perp}
$$


where $\varphi_{0}^{i}, \tilde{\varphi}_{0}^{i} \in \mathbb{E}_{0}=\mathbb{R}$ are constant functions on $S^{1}, \varphi_{1}^{i}, \tilde{\varphi}_{1}^{i}$ belong to $\mathbb{E}_{1}=$ $\operatorname{ker}\left(\Delta_{S^{1}}+1\right)=\operatorname{Span}\left\{e_{1}, e_{2}\right\}$ and where $\varphi^{i, \perp}, \tilde{\varphi}^{i, \perp}$ are $L^{2}\left(S^{1}\right)$ orthogonal to $\mathbb{E}_{0}$ and $\mathbb{E}_{1}$.

Projecting the Eqs. (49) over $\mathbb{E}_{0}$ will yield the system

$$
\left\{\begin{array}{l}
-2 \log \tau_{i}+4 \tilde{\eta}^{i} \log r_{\varepsilon, \lambda}-\mathcal{F}_{i}\left(\tilde{x}_{i}, \tilde{\mathbf{x}}\right)+\mathcal{O}\left(r_{\varepsilon, \lambda}^{2}\right)=0 \\
4 \tilde{\eta}^{i}+\mathcal{O}\left(r_{\varepsilon, \lambda}^{2}\right)=0 .
\end{array}\right.
$$

Let us comment briefly how these equations are obtained. They simply come from (49) when expansions (46) and (47) are used, together with the expression of $H^{i}$ and $H^{e}$ given in Lemmas 2 and 3, and also the estimates (43) and (44). The system (50) can be readily simplified into

$$
\frac{1}{\log r_{\varepsilon, \lambda}}\left[2 \log \tau_{i}+\mathcal{F}_{i}\left(\tilde{x}_{i}, \tilde{\mathbf{x}}\right)\right]=\mathcal{O}\left(r_{\varepsilon, \lambda}^{2}\right) \quad \text { and } \quad \tilde{\eta}^{i}=\mathcal{O}\left(r_{\varepsilon, \lambda}^{2}\right) .
$$

We are now in a position to define $\tau_{-}$and $\tau^{+}$since, according to the above, as $\varepsilon$ and $\lambda$ tend to 0 we expect that $\tilde{x}_{i}$ will converge to $x_{i}$ and that $\tau_{i}$ will converge to $\tau_{i}^{*}$ satisfying

$$
2 \log \tau_{i}^{*}=-\mathcal{F}_{i}\left(x_{i}, \mathbf{x}\right)
$$

and hence it is enough to choose $\tau_{-}$and $\tau^{+}$in such a way that

$$
2 \log \left(\tau_{-}\right)<-\sup _{i} \mathcal{F}_{i}\left(x_{i}, \mathbf{x}\right) \leq-\inf _{i} \mathcal{F}_{i}\left(x_{i}, \mathbf{x}\right)<2 \log \left(\tau^{+}\right) .
$$

We now consider the $L^{2}$-projection of $(49)$ over $\mathbb{E}_{1}$. Given a smooth function $f$ defined in $\Omega$, we identify its gradient $\nabla f=\left(\partial_{x_{1}} f, \partial_{x_{2}} f\right)$ with the element of $\mathbb{E}_{1}$

$$
\bar{\nabla} f=\sum_{i=1}^{2} \partial_{x_{i}} f e_{i}
$$

With these notations in mind, we obtain the equations

$$
\bar{\nabla} \mathcal{F}_{i}\left(\tilde{x}_{i}, \tilde{\mathbf{x}}\right)=\mathcal{O}\left(r_{\varepsilon, \lambda}^{2}\right) \quad \text { and } \quad \varphi_{1}^{i}=\mathcal{O}\left(r_{\varepsilon, \lambda}^{2}\right)
$$

Finally, we consider the $L^{2}$-projection onto $L^{2}\left(S^{1}\right)^{\perp}$. This yields the system

$$
\left\{\begin{array}{l}
\varphi^{i, \perp}-\tilde{\varphi}^{i, \perp}+\mathcal{O}\left(r_{\varepsilon, \lambda}^{2}\right)=0 \\
\partial_{r}\left(H^{i, \perp}-H^{e, \perp}\right)+\mathcal{O}\left(r_{\varepsilon, \lambda}^{2}\right)=0 .
\end{array}\right.
$$

Thanks to the result of Lemma 4, this last system can be re-written as

$$
\varphi^{i, \perp}=\mathcal{O}\left(r_{\varepsilon, \lambda}^{2}\right) \quad \text { and } \quad \tilde{\varphi}^{i, \perp}=\mathcal{O}\left(r_{\varepsilon, \lambda}^{2}\right) .
$$

If we define the parameters $\mathbf{t}=\left(t_{i}\right) \in \mathbb{R}^{m}$ by

$$
t_{i}=\frac{1}{\log r_{\varepsilon, \lambda}}\left[2 \log \tau_{i}+\mathcal{F}_{i}\left(\tilde{x}_{i}, \tilde{x}\right)\right], \quad \forall 1 \leq i \leq m,
$$

then the system we have to solve reads

$$
\left(\mathbf{t}, \tilde{\eta}, \varphi_{0}, \tilde{\varphi}_{0}, \varphi_{1}, \tilde{\varphi}_{1}, \bar{\nabla} \mathcal{F}(\tilde{\mathbf{x}}, \mathbf{x}), \varphi^{\perp}, \tilde{\varphi}^{\perp}\right)=\mathcal{O}\left(r_{\varepsilon, \lambda}^{2}\right),
$$

where as usual, the term $\mathcal{O}\left(r_{\varepsilon, \lambda}^{2}\right)$ depends nonlinearly on all the variables on the left side, but is bounded (in the appropriate norm) by a constant (independent of $\varepsilon$ and $\lambda$ ) time $r_{\varepsilon, \lambda}^{2}$, provided $\varepsilon \in\left(0, \varepsilon_{\kappa}\right)$ and $\lambda \in\left(0, \lambda_{\kappa}\right)$. Then the nonlinear mapping which appears on the right-hand side of (53) is continuous and 
compact. In addition, reducing $\varepsilon_{\kappa}$ and $\lambda_{\kappa}$ if necessary, this nonlinear mapping sends the ball of radius $\kappa r_{\varepsilon, \lambda}^{2}$ (for the natural product norm) into itself, provided $\kappa$ is fixed large enough. Applying Schauder's fixed Theorem in the ball of radius $\kappa r_{\varepsilon, \lambda}^{2}$ in the product space where the entries live yields the existence of a solution of Eq. (53) and this completes the proof of Theorem 1.

\section{Acknowledgments}

This article is supported by College of Science of King Saud University, Research Center project No. Math/2010/31.

\section{References}

[1] Bandle, C., Giarrusso, E.: Boundary blowup for semilinear elliptic equations with nonlinear gradient terms. Adv. Differ. Equ. 1, 133-150 (1996)

[2] Baraket, S., Ben Omrane, I., Ouni, T., Trabelsi, N.: Singular limits for 2-dimensional elliptic problem with exponentially dominated nonlinearity and singular data. Preprint

[3] Baraket, S., Dammak, M., Ouni, T., Pacard, F.: Singular limits for a 4-dimensional semilinear elliptic problem with exponential nonlinearity. Ann. I. H. Poincaré - AN 24, 875-895 (2007)

[4] Baraket, S., Pacard, F.: Construction of singular limits for a semilinear elliptic equation in dimension 2. Calc. Var. Partial Differ. Equ. 6, 1-38 (1998)

[5] Del Pino, M., Kowalczyk, M., Musso, M.: Singular limits in Liouville type equations. Calc. Var. Partial Differ. Equ. 24, 47-81 (2005)

[6] Esposito, P., Grossi, M., Pistoia, A.: On the existence of Blowing-up solutions for a mean field equation. Ann. I. H. Poincaré - AN 22, 227-257 (2005)

[7] Giarrusso, E.: Asymptotic behavior of large solutions of an elliptic quasilinear equation with a borderline case. C. R. Acad. Sci. Paris, Ser. I. 331, 777-782 (2000)

[8] Giarrusso, E.: On blow up solutions of a quasilinear elliptic equation. Math. Nachr. 213, 89-104 (2000)

[9] Greco, A., Porru, G.: Asymptotic estimates and convexity of large solutions to semilinear elliptic equations. Differ. Integral Equ. 10, 219-229 (1997)

[10] Liouville, J.: Sur l'équation aux différences partielles $\partial^{2} \log \frac{\lambda}{\partial u \partial v} \pm \frac{\lambda}{2 a^{2}}=0$. J. Math. 18, 17-72 (1853)

[11] Marcus, M., Véron, L.: Uniqueness of solutions with blowup on the boundary for a class of nonlinear elliptic equations. C. R. Acad. Sci. Paris, Ser. I. 317, $557-563$ (1993)

[12] Osserman, R.: On the inequality $\Delta u \geq f(u)$. Pac. J. Math. 7, 1641-1647 (1957) 
[13] Ren, X., Wei, J.: On a two-dimensional elliptic problem with large exponent in nonlinearity. Trans. Am. Math. Soc. 343, 749-763 (1994)

[14] Suzuki, T.: Tow dimensional Emden-Fowler equation with exponential nonlinearity. In: Nonlinear Diffusion Equations and Their Equilibrium States 3, pp. 493-512. Birkäuser (1992)

[15] Tao, S., Zhang, Z.: On the existence of explosive solutions for semilinear elliptic problems. Nonlinear Anal. 48, 1043-1050 (2002)

[16] Tarantello, G.: On Chern-Simons theory. In: Berestycki, H. (ed.) Nonlinear PDE's and Physical Modeling: Superfluidity, Superconductivity and Reactive Flows. Kluwer Academic Publisher, Dordrecht (2002)

[17] Wente, H.C.: Counter example to a conjecture of H. Hopf. Pac. J. Math. 121, 193-243 (1986)

[18] Wei, J., Ye, D., Zhou, F.: Bubbling solutions for an anisotropic Emden-Fowler equation. Calc. Var. Partial Differ. Equ. 28(2), 217-247 (2007)

[19] Weston, V.H.: On the asymptotique solution of a partial differential equation with exponential nonlinearity. SIAM J. Math. 9, 1030-1053 (1978)

[20] Ye, D., Zhou, F.: A generalized two dimensional Emden-Fowler equation with exponential nonlinearity. Calc. Var. Partial Differ. Equ. 13, 141-158 (2001)

[21] Zhang, Z., Tao, S.: On the existence and asymptotic behavior of explosive solutions for semilinear elliptic problems. Acta Math. Sin. 45A, 493-700 (2002) (in Chinese)

[22] Zhao, C.: Blowing-up solutions to an anisotropic Emden-Fowler equation with a singular source. J. Math. Anal. Appl. 342, 398-422 (2008)

\section{S. Baraket}

Department of Mathematics, College of Science,

King Saud University,

P.O. Box 2455,

Riyadh 11451,

Saudi Arabia

e-mail: sbaraket@ksu.edu.sa; smbaraket@yahoo.fr

I. Ben Omrane, T. Ouni

Département de Mathématiques, Faculté des Sciences de Tunis,

Campus Universitaire

2092 Tunis,

Tunisia

e-mail: benomraneines@gmail.com

T. Ouni

e-mail: Taieb.Ouni@fst.rnu.tn

Received: 07 June 2009.

Accepted: 16 July 2010. 